\title{
Nucleosomes as treadmills
}

\author{
Chromatin remodelers couple inchworm motion with twist-defect \\ formation to slide nucleosomal DNA
}

Authors: Giovanni B. Brandani, Shoji Takada

PlOS Comput Biol 14, e1006512 (2018).

\section{Recommended with a Commentary by Helmut Schiessel, Leiden University}

Active matter is currently very popular among physicists. But chromatin remodellers, molecular machines that act on our genetic material, have been widely neglected. The reason might be that we do not understand well what remodellers do and how they work. Remarkably there has now come some movement into the field by freezing chromatin remodellers.

Before discussing the experiment [1] and the ensuing computer simulation by Brandani and Takada I need to explain how our DNA (and that of all eukaryotes including animals, plants and fungi) is organised. DNA molecules can be centimetres long but need to fit into micron sized nuclei. This is achieved by packing them with proteins in a hierarchical fashion into the chromatin complex. We focus here on the first level, the wrapping of the DNA double helix around millions of protein cylinders. The resulting DNA spools are called nucleosomes, each wrapping 147 base pairs. The positions of the nucleosomes on the DNA are important as DNA wrapped into a nucleosome is inaccessible to most DNA binding proteins. The cell has therefore a set of motor proteins at its disposal to modify and to move nucleosomes along the DNA. These motor proteins are the chromatin remodellers.

How do they work? Especially, how can they move nucleosomes along DNA? One of the problems is that DNA inside a nucleosome is bound at discrete spots to the protein cylinder and that the total binding energy amounts to many tens of $k_{B} T$. Remodellers use ATP and have therefore much less energy at their disposal. They cannot act in groups on nucleosomes (like myosin in muscles) as there is simply not enough space for more than one remodeller to push or pull a nucleosome along.

A breakthrough has been achieved by transmission electron cryomicroscopy (cryo-EM), a field that has recently made huge steps forward. In this particular case, a remodeller (Snf2) in complex with a nucleosome has been studied [1]. By processing cryo-EM data from about 200000 of these particles, their structure could be determined with about $5 \AA$ resolution. It was found that the remodeller is bound asymmetrically on the nucleosome, namely at a location about 20 basepairs away from the middle of the wrapped portion, and that there is another contact with a stretch of DNA on the other nucleosomal turn.

What can one learn from the cryo-EM pictures about how the remodeller works, especially how it moves the nucleosome along the DNA? Here the beautiful molecular dynamics study 
by Brandani and Takada comes into play. The basis for this study is a coarse grained nucleosome model. Two groups have independently developed very similar models [2, 3] where one group (at the University of Chicago) provided the coarse-grained DNA model and the other (at Kyoto University) the coarse grained protein model. The resulting nucleosome models of both groups combine these two coarse grained representations. In these models the DNA double helix (which carries charges along its two helical backbones) is attracted to the oppositely charged protein cylinder via a Debye-Hückel interaction. As a result a nucleosome complex forms but the position of the cylinder along the DNA is not set by the simulation. Interestingly, both groups reported "sliding" of the protein cylinder along the DNA molecule. This phenomenon is known experimentally for a long time [4] but the simulations $[2,3]$ could finally unravel the underlying physical mechanism. It was found that the mobility of the model nucleosomes is caused by defects, typically small twist defects that form inside the nucleosome or come in from the ends. These defects consist of a missing or an extra basepair. They cause the repositioning of nucleosomes by one base pair steps.

Could the chromatin remodellers mobilise nucleosomes by actively creating defects inside nucleosomes? How this could work is far from obvious. The remodeller contains a motor that shares similarities with a class of well-known motors called helicases. Some helicases move unidirectionally along DNA essentially in an inchworm fashion. To achieve this they feature two lobes that bind to the DNA, changing their distance and modulating individually their binding strengths through each ATP cycle. As a result they walk like we would walk, if we would keep always the same foot in front of the other one. However, whereas the helicase moves along following the DNA helix, a remodeller bound to a nucleosome cannot perform any motion as it would clash either with the protein core or (moving in the opposite direction) with DNA from the other nucleosomal turn. So how can a remodeller move a nucleosome along DNA?

Brandani and Takada used the information from the cryo-EM structure of the nucleosomeremodeller complex [1] to place the remodeller asymmetrically onto their nucleosome model. Using the similarity to helicases, they let their remodeller model go through three chemical states where each has a slightly different force field. When the authors led their remodeller act on bare DNA, not surprisingly it walked along the double helix in an inchworm fashion. About 50\% of the ATP cycles led to successful steps. Remarkably, the remodeller bound to the nucleosome managed to walk along the DNA as well, even though it seems to have no space to move. Even more remarkable is the fact that it managed to drag the whole nucleosome along and practically each cycle led to a successful step.

How does the remodeller executes this seemingly impossible feat? In the starting position (the one corresponding to the cryo-EM structure [1]) the two lobes are distant. ATP binding leads to the motion of one lobe toward the other, changing the remodeller from an open to a closed configuration. As a result there is now a gap left behind that lobe. Surprising is what happens next: the remodeller (still in the closed configuration) swings back, closing the gap and deforming with it the DNA around the position where it is bound. What causes this motion is a basic patch on the lobe that is attracted to the other DNA turn with which it was in contact with before. Next, ATP hydrolysis can take place as there is now space for the other lobe to move so that the remodeller can go back to the open configuration and the cycle is finished.

Now we can return to the twist defects. During the swinging-back of the remodeller, 
caused by the "electrostatic spring", the DNA is deformed. As a result, a twist defect with an extra base pair has formed on one side of the remodeller (between the two closest binding sites of the DNA to the protein cylinder) and another one with a missing base pair on the other side. With the electrostatic spring holding the remodeller tightly in place, this over/ under-twist pair cannot annihilate itself. Instead the defects diffuse along their respective wrapped DNA portions and fall off at the ends. As a result the nucleosome makes a step by one base pair. The whole scenario reminds me of historical treadmills (or treadwheels), machines where humans provided the power. Instead of the electrostatic spring pulling the remodeller back, it was gravity that pulled the person after each step down again, causing the rotation of the wheel.

As we are now starting to understand how remodellers work on the microscopic scale, new exciting questions can be addressed. One that I find especially interesting is how the positions of the nucleosomes along DNA are affected by the action of the remodellers. It is known that nucleosomes have certain sequence preferences [5] which reflect the fact that the elasticity and shape of the DNA double helix depends on the underlying sequence of base pairs. In the absence of remodellers (e.g. in the test tube) nucleosomes reposition themselves in the resulting free energy landscape. However, for longer DNA molecules this thermally induced repositioning, i.e. the above mentioned nucleosome sliding, is too slow to achieve equilibration on any meaningful time scale. Remodellers speed up this process but might at the same time cause nucleosomes to end up on positions far from equilibrium. This is especially interesting, as the internal energy landscape of the remodeller shows a strong sequence dependence, as Brandani and Takada noticed. To understand where nucleosomes end up along the free energy landscape provided by the DNA, under the action of chromatin remodellers, remains an exciting unsolved problem.

\section{References}

[1] X. Liu, M. Li, X. Xia, X. Li, Z. Chen, Nature 544, 440 (2017).

[2] J. Lequieu, D. C. Schwartz, J. J. de Pablo, PNAS 114, E9197 (2017).

[3] G. B. Brandani, T. Niina, C. Tan and S. Takada, Nucl. Acids Res. 46, 2788 (2018).

[4] G. Meersseman, S. Pennings, E. M. Bradbury, EMBO J. 11, 2951 (1992).

[5] N. Kaplan, I. K. Moore, Y. Fondufe-Mittendorf, A. J. Gossett, D. Tillo, Y. Field, E. M. LeProust, T. R. Hughes, J. D. Lieb, J. Widom, E. Segal, Nature 458, 362 (2009). 